\title{
Article \\ Characterization of a Novel Double-Stranded RNA Virus from Phytophthora pluvialis in New Zealand
}

\author{
Zhi Xu ${ }^{1,2} \mathbb{E}^{\mathbb{D}}$, Mahmoud E. Khalifa ${ }^{3,+} \mathbb{D}$, Rebekah A. Frampton $^{1} \mathbb{D}$, Grant R. Smith ${ }^{1} \mathbb{D}$, Rebecca L. McDougal $^{4} \mathbb{D}$, \\ Robin M. MacDiarmid 2,3 and Falk Kalamorz ${ }^{1, *}$
}

check for

updates

Citation: Xu, Z.; Khalifa, M.E.;

Frampton, R.A.; Smith, G.R.;

McDougal, R.L.; MacDiarmid, R.M.;

Kalamorz, F. Characterization of a

Novel Double-Stranded RNA Virus

from Phytophthora pluvialis in New

Zealand. Viruses 2022, 14, 247.

https: / doi.org/10.3390/v14020247

Academic Editors: Vernon Ward

and John A. Taylor

Received: 15 December 2021

Accepted: 12 January 2022

Published: 26 January 2022

Publisher's Note: MDPI stays neutral with regard to jurisdictional claims in published maps and institutional affiliations.

Copyright: () 2022 by the authors Licensee MDPI, Basel, Switzerland. This article is an open access article distributed under the terms and conditions of the Creative Commons Attribution (CC BY) license (https:// creativecommons.org/licenses/by/ $4.0 /)$.

1 The New Zealand Institute for Plant and Food Research Limited, Canterbury Agriculture \& Science Centre, 74 Gerald Street, Lincoln 7608, New Zealand; Zhi.Xu@plantandfood.co.nz (Z.X.);

Rebekah.Frampton@plantandfood.co.nz (R.A.F.); Grant.Smith@plantandfood.co.nz (G.R.S.)

2 School of Biological Sciences, University of Auckland, Auckland 1142, New Zealand; Robin.MacDiarmid@plantandfood.co.nz

3 The New Zealand Institute for Plant and Food Research Limited, 120 Mt Albert Road, Sandringham, Auckland 1025, New Zealand; mkha201@aucklanduni.ac.nz

4 Scion, New Zealand Forest Research Institute, Ltd., 49 Sala Street, Te Papa Tipu Innovation Park, Rotorua 3010, New Zealand; Rebecca.McDougal@scionresearch.com

* Correspondence: Falk.Kalamorz@plantandfood.co.nz

† Current address: Department of Botany and Microbiology, Faculty of Science, Damietta University, Damietta 34511, Egypt.

\begin{abstract}
A new dsRNA virus from the oomycete Phytophthora pluvialis has been characterized and designated as Phytophthora pluvialis RNA virus 1 (PplRV1). The genome of the PplRV1 reference genome is $6742 \mathrm{bp}$ that encodes two predicted open reading frames (ORFs). ORF1 and ORF2 overlap by a 47 nt "slippery" frameshift sequence. ORF1 encodes a putative protein of unknown function. ORF2 shows high similarity to the RNA-dependent RNA polymerase (RdRp) of other dsRNA viruses. Phylogenetic analysis of the putative PplRV1 RdRp and its most closely related viruses showed PplRV1 is distinct from other known viruses (below 33\% amino acid similarity), which indicates this virus may belong to a new virus family. Analyses of the geographical distribution of PplRV1 in relation to two genetically distinct classes of its host revealed two corresponding genotypes of the PplRV1 (termed a and b), which share 92.3\% nt identity. The reference genome for the second genotype is $6760 \mathrm{bp}$ long and a prediction of its genetic organization shows three ORFs, with ORF2 being split into two ORFs, ORF2a and ORF2b, that is conserved in seven of eleven genotype $b$ isolates. Additionally, a quick and simple diagnostic method using qPCR has been developed, which is suitable for large scale screens to identify PplRV1 in Phytophthora.
\end{abstract}

Keywords: virus; Phytophthora pluvialis; dsRNA; qPCR; New Zealand

\section{Introduction}

Phytophthora is a genus in the family Oomycota, which was previously classified within the fungi domain and separated owing to their highly divergent genome sequence [1]. Most Phytophthora species are plant pathogens and cause enormous economic losses in crops worldwide. The most notorious plant pathogen, Phytophthora infestans, causes potato late blight which in the 1840s triggered a series of events that led to several million people dying from starvation [2]. Another example is Phytophthora agathidicida, the causal agent of the devastating disease kauri dieback which threatens kauri tree (Agathis australis) populations of high ecological and cultural relevance in New Zealand [3-5].

Phytophthora pluvialis is a pathogen causing red needle cast disease. In 2008, a severe needle disease of Pinus radiata caused by a new and undescribed Phytophthora was reported in New Zealand [6]. In 2013, P. pluvialis was isolated from stream, soil and canopy drip in tanoak-Douglas fir forest in Oregon (USA) and described as a new pathogen species within 
the Phytophthora clade III [7]. A year later, P. pluvialis was first recognized in the North Island of New Zealand when an outbreak of red needle cast disease on pine trees (Pinus radiata) occurred $[6,8]$.

A study using single nucleotide polymorphism (SNP) and a minimum spanning network identified two clusters of P. pluvialis isolates from New Zealand, which suggested $P$. pluvialis was potentially introduced from the USA into New Zealand on two separate occasions [9]. A subsequent comparison with isolates from the USA using a larger range of SNP markers showed that one of these New Zealand clusters is closely related to isolates from the USA, while the other may represent an evolutionary adaptation to the new environment [10].

Increasing numbers of viruses have been reported in Phytophthora since the first virus was identified in 2005. Originally, an endornavirus was isolated from a Phytophthora isolate from Douglas fir [11] and named Phytophthora endornavirus 1 (PEV1). The genome of PEV1 is $13,883 \mathrm{nt}$, and contains an open reading frame (ORF) encoding a potential RNA-dependent RNA polymerase (RdRp) [11]. PEV1 has not only been confirmed in a Phytophthora isolate from Douglas fir but also in Phytophthora ramorum [12]. In 2020, a dsRNA virus was discovered infecting Phytophthora cactorum and designated Phytophthora cactorum RNA virus 1 (PcRV1) [13]. Phylogenetic analysis reveals this virus is closely related to the unclassified Pythium splendens RNA virus 1, which is provisionally classified into the virus family Totiviridae [14]. To date there are seven virus species recognized within Phytophthora [11-13,15-18].

Several RNA viruses have been found in single Phytophthora species. For example, within Phytophthora infestans alone four viruses have been described. An RNA virus was reported in P. infestans and designated Phytophthora infestans RNA virus 1 (PiRV-1) [15]. PiRV-1 has two RNA segments, RNA1 and RNA2, with a length of $3160 \mathrm{nt}$ and $2730 \mathrm{nt}$, respectively. RNA1 encodes a hypothetical protein and an RdRp. RNA2 is predicted to encode a polyprotein and an ORF of unknown function. Phylogenetic analysis reveals PiRV-1 does not belong to any known virus family [15]. Another RNA virus, Phytophthora infestans RNA virus 2 (PiRV-2), was discovered in P. infestans in 2018 [18]. The genome of PiRV-2 is $11,170 \mathrm{nt}$, which contains a single predicted ORF spanning the nucleotides 7 to 11,139 . It encodes a putative polyprotein that functions as an $\operatorname{RdRp}$ [18]. The positive sense, single strand RNA (ssRNA) virus Phytophthora infestans RNA 3 (PiRV-3) was first been reported in 2013 and has a 8112 nt long genome that has two predicted ORFs with a $31 \mathrm{bp}$ overlap. The ORF1 of PiRV-3 potentially encodes a protein with an unknown function and ORF2 putatively encodes an RdRp [17]. In 2012, another virus named Phytophthora infestans RNA virus 4 (PiRV-4) was reported by the same group [16]. The genome of PiRV-4 is small, only $2984 \mathrm{nt}$. A single predicted ORF in the PiRV-4 genome potentially encodes an RdRp. A phylogenetic analysis of the PiRV-4 RdRp shows it is related to the positive sense ssRNA narnaviruses [16].

Beside RNA viruses, DNA viruses have also been reported in Phytophthora species. In 2011, Liu et al. found gemini-like sequences integrated into the mitochondrial genomes of P. infestans and Phytophthora sojae [19]. The sequence integrated into the P. infestans mitogenome shares approximately $70 \%$ similarity with the one found in $P$. sojae. It has been assumed that the replication-initiation sequences of some circular DNA viruses, such as geminiviruses, are frequently transferred into a wide variety of eukaryotic hosts [19]. Furthermore, several of the integrated genes are maintained and expressed in the host genomes [19].

Viruses or viral-like sequences can contribute to host-adaptive phenotypes, including thermal tolerance and virulence. For example, Dichanthelium lanuginosum, a tropical panic grass, colonized by the fungus Curvularia protuberata which itself is infected with the C. protuberata thermal tolerance virus, is more heat-tolerant than plants harboring virusfree C. protuberata [20]. A group of mycoviruses were found to be able to decrease the infectivity of their pathogenic fungal host, such as Cryphonectria hypovirus 1 (CHV1) infesting Cryphonectria parasitica, which causes chestnut blight [21,22]. Because CHV1 is 
also associated with an increase in the survival time of host plants, this mycovirus was used as a biocontrol agent of chestnut blight in Europe [21]. Additionally, study revealed PiRV-2 affects the sporulation in P. infestans [23]. Compared to PiRV-2-free isogenic isolates, $P$. infestans isolates that contained PiRV-2 produced a larger number sporangia. Further surveys found PiRV-2 was present in most strains of a predominant clonal lineage of P. infestans in North America, which suggested PiRV-2 could be the cause of the resurgence of potato late blight in the 1980s-1990s [23]. Mycoviruses that reduce the pathogenicity of their fungal host are known as hypoviruses. They have the ability to decrease the chance of infection, colonization and/or death by the pathogenic fungus. Conversely, viruses that increase the infectivity of fungi are called hyperviruses and these are typically associated with reduced survival time of infected plants [24].

Understanding of the potential impacts of Phytophthora viruses on Phytophthora, either hypervirulence or hypovirulence, may lead to alternative options to control pathogenic Phytophthora. This study discovered and characterized an RNA virus associated with New Zealand P. pluvialis, which provides fundamental knowledge and may identify leads for developing a biocontrol method to manage pathogenic P. pluvialis.

\section{Methods}

\subsection{Initial Screen for Phytophthora dsRNA Elements}

An initial screen for double-stranded RNA elements was performed with isolates NZFS 4014 to 4019 collected in Gisborne, New Zealand, using the CF-11 cellulose chromatography protocol [25], with subsequent analysis on 1\% SDS-Agarose gels. The extracted dsRNA was purified as described by Valverde et al. (1990). Illumina sequencing and bioinformatics analysis were then performed as described in Khalifa et al. (2016).

\subsection{P. pluvialis Isolates for Virus Genome Characterization}

P. pluvialis isolates collected in the New Zealand regions of Auckland ( $\mathrm{n}=1)$, Bay of Plenty/Wanganui $(n=1)$, Coromandel $(n=2)$, Gisborne $(n=7)$, Hawke's Bay $(n=1)$, Nelson $(n=3)$, Northland $(n=5)$, Taranaki $(n=1)$, Taupo $(n=1)$ and Wairarapa $(n=1)$ were obtained from the New Zealand Forest Research Institute (Scion) (Rotorua, New Zealand). Among these P. pluvialis, two are isolates whose cluster is unknown, 12 isolates belong to New Zealand cluster 1 (NZ1), and nine isolates belong to New Zealand cluster 2 (NZ2) [9]. Moreover, one isolate was isolated from Douglas-fir (Pseudotsuga menziesii), and 22 isolates were sourced from radiata pine (Pinus radiata) (Table S1). Isolates were maintained on V8 agar [26] supplemented with $250 \mathrm{mg} / \mathrm{L}$ ampicillin (AppliChem GmbH, Darmstadt, Germany), $10 \mathrm{mg} / \mathrm{L}$ natamycin (Sigma-Aldrich ${ }^{\circledR}$, St. Louis, MO, USA) and $250 \mathrm{mg} / \mathrm{L}$ rifampicin (Sigma-Aldrich ${ }^{\circledR}$, St. Louis, MO, USA) at room temperature in the dark.

\section{3. dsRNA Extraction and Sequencing}

After 12-14 days, 100-400 mg Phytophthora mycelia were collected for dsRNA extraction. Mycelia were ground in liquid nitrogen using a sterilized pestle and mortar. The dsRNA was extracted using Double-RNA Viral dsRNA Extraction Mini Kit (ABC Scientific ${ }^{\circledR}$, Glendale, CA, USA) according to the manufacturer's instructions for plant tissue.

Virus dsRNA was reverse-transcribed into complementary DNA (cDNA) with primer SEQ31 designed based on the viral sequences initially isolated using SuperScript ${ }^{\mathrm{TM}}$ IV First-Strand cDNA Synthesis Reaction Kit (Invitrogen, Carlsbad, CA, USA). Subsequently, it was amplified via Polymerase chain reaction (PCR) with primers SEQ31 and SEQ6663 (Table S2). The full-length sequence of this cDNA was obtained by primer walking and $5^{\prime}$ rapid amplification of cDNA ends (5'RACE). Virus genomes from isolates NZFS 3052 and NZFS 4018 were chosen as reference for PplRV1 from P. pluvialis clusters NZ1 and NZ2, respectively [10]. To obtain the terminal sequences, $5^{\prime}$ RACE was performed on virus dsRNA. Virus dsRNA was reverse-transcribed into cDNA by reverse transcriptase (RT) and the RACE1 primer (Table S2). A poly (A) tail was then added to the cDNA ends using terminal deoxynucleotide transferase (New England Biolabs, Ipswich, MA, USA). The 
polyadenylated cDNA fragment was amplified by the anchor primer and primer RACE2. Terminal sequences were finally amplified by the adaptor primer and primer RACE3. The size and quality of the resulting products were checked by $1 \%$ agarose gel electrophoresis and NanoDrop ${ }^{\mathrm{TM}} 1000$ Spectrophotometer (Thermo Fisher Scientific, Madison, WI, USA).

The purified PCR products were sequenced via a commercial Sanger sequencing service (Massey University Genome Sequencing Service, Palmerston North, New Zealand). Subsequently a sequencing strategy using eight distinct overlapping PCR was established (see Table S2 for details). Virus dsRNA from 23 P. pluvialis isolates were fully/near completely sequenced.

\subsection{Bioinformatic Analysis}

Low-quality ends of the Sanger sequences amplified were trimmed and de novo assembled using Geneious 10.0.9 (Biomatters, Auckland, New Zealand). The sequence assembly were aligned to the preliminary contigs 1 and 2 assembled from the initial screen. The ORFs in the virus genome were predicted by Geneious 10.0.9.

Virus genomes from NZFS 3052 and NZFS 4018 were used as the references for virus sequence assembly from additional P. pluvialis isolates. Virus genomes from P. pluvialis isolates belonging to NZ1 and NZ2 [10] were aligned using MAFFT [27,28] via Geneious 10.0.9 to identify any cluster-specific single-nucleotide polymorphisms (SNPs). The nucleotide alignment were used to build a phylogenetic trees using PhyML 3.0 [29,30] via Geneious 10.0.9.

A conserved region within the virus genome was found through NCBI Conserved Domain Database [31] searches. BLASTx search of the predicted conserved region was performed. Amino acid sequences of the putative conserved region and its related viruses, including Wuhan insect virus 28 (accession: YP_009342430), Circulifer tenellus virus 1 (accession: YP_003800003), Spissistilus festinus virus 1 (accession: YP_003800001), Culex vishnui subgroup totivirus (accession: BBQ05098), Stinn virus (accession: QRW41701), Koroku virus (accession: QRW41695), Hubei toti-like virus 10 (accession: YP_009336493), Phytophthora infestans RNA virus 3 (accession: YP_009551328) and Bremia lactucae associated fusagravirus1 (accession: QIP68009) were then aligned using MAFFT [27,28]. Additionally, an alignment of the amino acid sequence of the putative conserved region within the virus genome was generated using Clustal Omega [32]. FSFinder 2 [33] was used to identify frameshifts within the virus genomes. The structure of the predicted slippery sequence was analyzed and visualized in Geneious 10.0.9.

\subsection{Virus Diagnostics}

A panel of Phytophthora/Nothophytophthora isolates was used for the virus screen, including 25 P. pluvialis isolates, nine other Phytophthora species/isolates and one Nothophytophthora species. The 25 P. pluvialis isolates were collected in Bay of Plenty $(\mathrm{n}=2)$, Gisborne $(n=1)$, Hawke's Bay $(n=3)$, Nelson $(n=5)$, Rangitikei $(n=3)$, Taranaki $(n=1)$, Taupo $(n=3)$, Waikato $(n=1)$, Wairarapa $(n=2)$, Wanganui $(n=1)$, Wellington $(n=3)$ in New Zealand. Two P. cactorum isolates were collected in the Bay of Plenty in New Zealand. Two Phytophthora cryptogea isolates were collected in the New Zealand regions of South Canterbury and Auckland. Two Phytophthora multivora isolates were collected in Auckland and the Bay of Plenty, and three Phytophthora hybrid isolates were collected in Auckland, the Bay of Plenty and Northland regions in New Zealand.

Phytophthora total RNA was extracted using RNeasy Mini Kit (Qiagen, Hilden, Germany) following the manufacturer's instructions. The RT- quantitative PCR (RT-qPCR) primers and probes were designed by Primer3 [34,35] via Geneious 10.0.9. A duplex RT-qPCR with primers PplRV1_qPCR_F1 and PplRV1_qPCR_R1 and probe PplRV1_probe1 for PplRV1 detection combined with primers PhyG-F2 and PhyG-Rb and probe TrnM_PhyG_probe2 as host control was used for detecting PplRV1 (see Table S2 for details). The RT-qPCR was performed using iTaq Universal Probes One-step kit (Bio-Rad, Hercules, CA, USA). Annealing temperature for qPCR amplification was optimized by gradient qPCR, followed 
by primer and probe optimization. The final concentration of primers PplRV1_qPCR_F1 and PplRV1_qPCR_R1 and probe PplRV1_probe1 were $400 \mathrm{nM} / 400 \mathrm{nM}$ and $250 \mathrm{nM}$, respectively. The final concentration of primers PhyG-F2 and PhyG-Rb and probe TrnM_PhyG_probe2 were $500 \mathrm{nM} / 500 \mathrm{nM}$ and $250 \mathrm{nM}$, respectively. An aliquot $(2 \mu \mathrm{L})$ of Phytophthora total RNA was used in each $20-\mu \mathrm{L}$ reaction. The optimized reactions are summarized in Table S3. The RT-qPCR cycling followed the manufacturer's instructions with the modification of annealing temperature at $55^{\circ} \mathrm{C}$. Total RNA was firstly reverse transcribed into cDNA at $50{ }^{\circ} \mathrm{C}$ for $10 \mathrm{~min}$, followed by $1 \mathrm{~min}$ of polymerase activation and DNA denaturation at $95^{\circ} \mathrm{C}$. Amplification consisted of 40 cycles of $10 \mathrm{~s}$ of denaturation at $95{ }^{\circ} \mathrm{C}$ and $30 \mathrm{~s}$ of annealing then extension at $55^{\circ} \mathrm{C}$. Fluorescence was read following the annealing and extension step of each cycle. Data analysis was conducted using Bio-Rad CFX Maestro ${ }^{\mathrm{TM}}$ Software (Bio-Rad, Hercules, CA, USA). The specificity and sensitivity of the assay was established using a panel of 35 Phytophthora/Nothophytophthora strains (Table S4).

\section{Results}

\subsection{Virus Sequence and Genome Characterisation}

Initial screen for Phytophthora dsRNA elements identified three viral contigs. The three contigs were assembled from dsRNA-derived cDNA sequences and translated in silico: contig 1 (3395 nt) and contig 2 (2865 nt) shared approximately 30\% amino acid identity with PiRV-3 hypothetical proteins and RdRp, respectively [17] while contig 3 (2957 nt) shared $31 \%$ amino acid identity with PiRV-4 [16].

The presence of a single viral dsRNA genome, designated as Phytophthora pluvialis RNA virus 1 (PplRV1), was confirmed by RT-PCR. The complete PplRV1 reference genome sequence was obtained through Sanger sequencing using primer walking and $5^{\prime} \mathrm{RACE}$ (Figure S1) from RNA extracted from isolate P. pluvialis NZFS 3052. The PplRV1_3052 genome is $6742 \mathrm{nt}$ with a GC content of $55.4 \%$, and two predicted ORFs. ORF1 is $3549 \mathrm{nt}$ in length, starting at nt position 455 and terminating at 4003. A BLASTx analysis of the predicted ORF1 amino acid sequence showed similarity to hypothetical proteins found in other RNA viruses, including PiRV-3. The ORF2 is 2631 nt in length, starting at position 4039 and terminating at position 6669. The ORF2 is predicted to encode an RdRp, and contains six predicted motif regions. A $47 \mathrm{nt}$ slippery sequence was predicted to span ORF1 and ORF2 from nt 3955 to nt 4001 (Figure 1A). A signal sequence for -1 translational frameshift, UUUAAAC, was identified immediately before the stop codon of ORF1. Moreover, a strong stem-loop structure forms in the slippery sequence and a six nt spacer region exists between the signal sequence and the stem-loop structure (Figure 1A). Together, these genomic signatures suggest that the two ORFs may be expressed as a fused protein.

To investigate the genetic diversity of PplRV1 in New Zealand P. pluvialis population, we isolated PplRV1 from a range of P. pluvialis isolates spanning both New Zealand clusters. Two distinct genotypes of PplRV1 (PplRV1a and b) were identified from the two host clusters (P. pluvialis NZ1 and NZ2) with complete correspondence, respectively. PplRV1 (genotype a) from isolate NZFS 3052 (P. pluvialis cluster NZ1) was chosen as the reference genome for the first genotype of the virus, and we propose to designate the virus genotype associated with NZFS 4018 (P. pluvialis cluster NZ2) as the reference genome for PplRV1b.

PplRV1_4018 has a genome size of $6760 \mathrm{nt}$ with a GC content of $55.2 \%$. Three ORFs including two sub-ORFs of ORF2 were identified in PplRV1_4018. ORF1 has a length of $3549 \mathrm{nt}$, starting at position 455 and terminating at 4003. ORF2a has a length of $1041 \mathrm{nt}$, which begins at position 4039 and ends at 5079. ORF2b is $1671 \mathrm{nt}$ in length, from position 5051 to position 6721 . Both ORF2a and ORF2b show similarity to parts of RdRp albeit encoded in two ORFs (Figure 1B). ORF2a and ORF2b contain different RdRp motifs, which are within six other PplRV1b isolates (refer to Section 3.3 for further details). Four PplRV1b isolates were sequenced that had the RdRp in a single ORF (ORF2). The separation of the RdRp into two ORFs is due to a change from a 'GGG' stretch to a 'GGGG' at position 5064 in PplRV1_4018. This insertion introduces a stop codon and terminates the predicted ORF. 


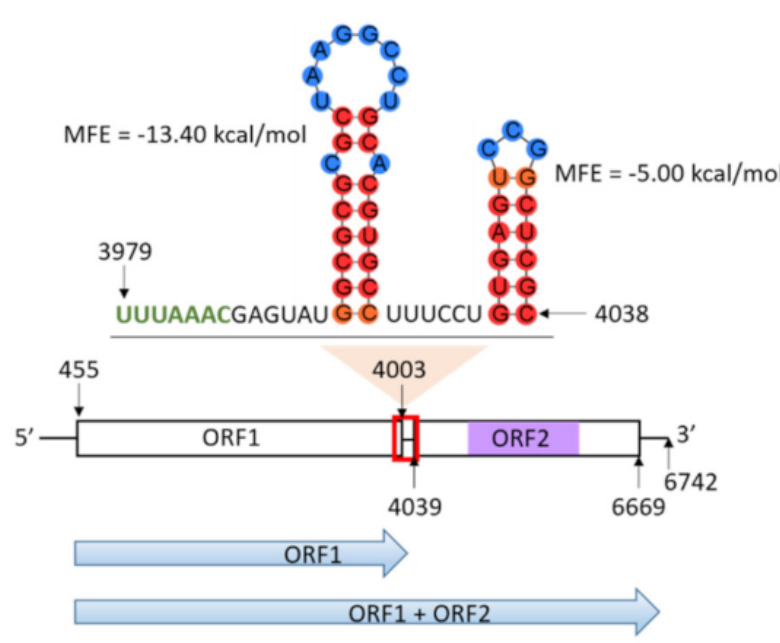

(A)

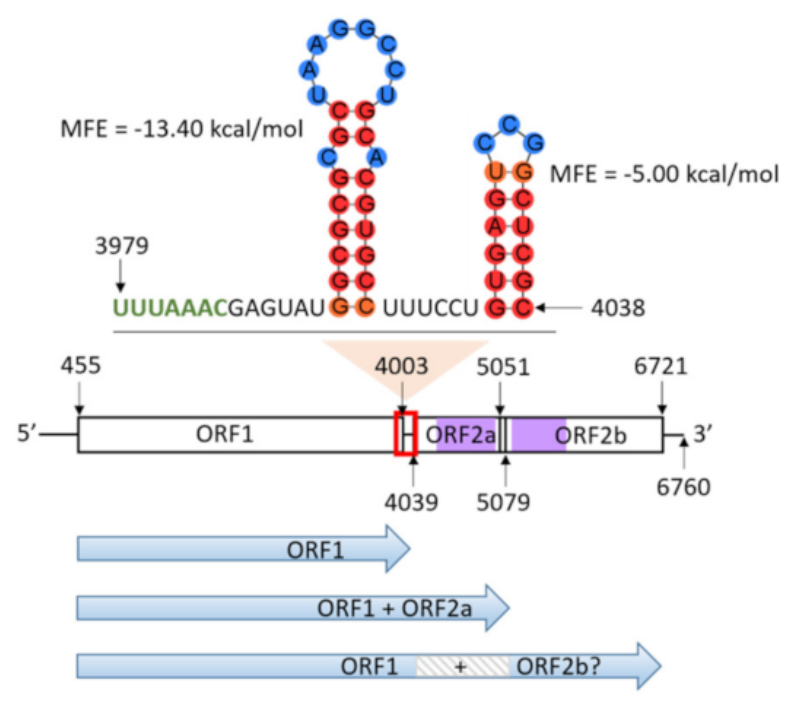

(B)

Figure 1. (A) Genome structure of Phytophthora pluvialis RNA virus 1 (PplRV1) genotype a from NZFS 3052. (B) Genome structure of PplRV1 genotype b from NZFS 4018. Horizontal bars represent open reading frames (ORFs). Purple bars represent RNA-dependent RNA polymerase (RdRp) regions where the conserved motifs are located. Lines represent the $5^{\prime}$ and $3^{\prime}$ ends and the gap sequence between ORF1 and ORF2. Nucleotide position in genomes are indicated by numbers. Red boxes indicate the position of the -1 reading frame signal sequence UUUAAAC (labeled in green), spacer region and downstream sequence with stem-loop structures. In the stem-loop structures, nucleotides in red/orange circular shades indicate high base-pairing probability. Nucleotides in blue circular shades indicate low base-pairing probability. Blue arrows represent possible protein translated from predicted ORFs. Grey box with diagonal lines indicates the protein that should be encoded by ORF2a. MFE: minimum free energy.

PplRV1a and PplRV1b share 92.3\% nt identity. Although PplRV1b has a different genome size and GC content from those of PplRV1a, it contains a perfectly conserved slippery sequence of the same size and in the same position and therefore identical stemloops (Figure 1A,B).

\subsection{PplRV1 Conserved Region and Related Viruses}

BLASTx searches using the protein sequence of the putative RdRp encoded by PplRV1a ORF2 found similarities with the RdRp regions of Wuhan insect virus 28 (WiV28), Circulifer tenellus virus 1 (CtV1), Spissistilus festinus virus 1 (SfV1), Culex vishnui subgroup totivirus (CvTV1), Stinn virus (SV), Koroku virus (KV), Hubei toti-like virus 10 (HTV10), Phytophthora infestans RNA virus 3 (PiRV-3), and Bremia lactucae associated fusagravirus 1 (BlaFV1). Within these related viruses, CvTV belongs to Totiviridae, and CtV1, SfV1 and BlaFV1 are dsRNA viruses, while WiV28, and SV, KV HTV10 and PiRV3 are unclassified RNA viruses. PplRV1a RdRp has very low residue/base similarity percentage with these other viruses. The highest amino acid similarity is $32.64 \%$ with dsRNA virus, BLaFV1, and the lowest amino acid similarity is $24.94 \%$ with unclassified RNA virus, SV (Table 1 ). Alignment of PplRV1a putative RdRp with WiV28, CtV1, SfV1, CvTV, SV, KV, HTV10, PiRV-3 and BlaFV1 RdRps revealed some conserved residues present among these proteins (Figure 2). Fully conserved residues at amino acid positions are present in the motifs of PplRV1 putative RdRp (Figure 2). As typical in viral RdRps, there are several conserved motifs [36]. In PplRV1a, six conserved motifs exist in the putative RdRp, including motif I (KYELAKNRVLW), motif II (DYSDFNINH), motif III (TGIRGTAFFNT), motif IV (GDD), motif V (GEFLR) and motif VI (GFLLR) (Figure 2), while in totivirus CvTV, motif I is KFEMGKSRAIYG; motif II is DYADFNYQH; motif III is SGFRGTNFLNT; motifs IV and V are GDD and GEFLR, respectively, and motif VI is GYLAR (Figure 2). Except for motifs IV 
and V, other motifs in PplRV1a and CvTV are different. The lack of conservation of motifs I, II, III and VI of PplRV1 RdRp indicates the virus replication mechanism of PplRV1 may differ from that of the totivirus, CvTV. Overall, the conserved region and phylogenetic analysis indicate that PplRV1a differs from its related viruses. Therefore, PplRV1 is likely to be a member of a so far unclassified virus family.

Table 1. Percentage identity of residues/bases among putative RNA-dependent RNA polymerase (RdRp) of Phytophthora pluvialis RNA virus 1 genotype a (PplRV1a) and RdRps of Culex vishnui subgroup totivirus (CvTV), Bremia lactucae associated fusagravirus1 (BlaFV1), Koroku virus (KV), Stinn virus (SV), Spissistilus festinus virus 1 (SfV1), Circulifer tenellus virus 1 (CtV1), Hubei toti-like virus 10 (HTV10), Wuhan insect virus 28 (WiV28) and Phytophthora infestans RNA virus 3 (PiRV-3).

\begin{tabular}{|c|c|c|c|c|c|c|c|c|c|c|}
\hline & PplRV1a & CvTV & BlaFV1 & KV & SV & SfV1 & CtV1 & HTV10 & WiV28 & PiRV-3 \\
\hline CvTV & 25.97 & & & & & & & & & \\
\hline BlaFV1 & 32.64 & 24.23 & & & & & & & & \\
\hline KV & 27.65 & 57.14 & 26.79 & & & & & & & \\
\hline SV & 24.94 & 69.42 & 24.74 & 53.28 & & & & & & \\
\hline SfV1 & 25.42 & 34.15 & 23.56 & 44.23 & 32.93 & & & & & \\
\hline $\mathrm{CtV} 1$ & 26.09 & 35.04 & 20.62 & 43.08 & 34.31 & 62.29 & & & & \\
\hline HTV10 & 25.71 & 55.32 & 24.23 & 60.62 & 53.19 & 35.85 & 34.55 & & & \\
\hline WiV28 & 27.62 & 25.77 & 24.37 & 30.22 & 22.96 & 24.05 & 23.52 & 22.70 & & \\
\hline PiRV-3 & 29.72 & 24.68 & 31.78 & 24.72 & 24.94 & 19.19 & 19.14 & 26.74 & 24.87 & \\
\hline
\end{tabular}

PpIRV1a MLSRMVDKYELAKNRVLWNSQVLHYMISCYLLEPIEHQL-GLLSELESYSESLNGLREQF WiV28 VLSTLVPKFENGKVRALSNTENWEYVLESYLLRGFEDTL-QEVEGALMKEGGLSEVAKIK CtV1 EVATASEKYENGKARAIYGVAPMHYVLNTYATKGFEERF-HLVEGLEKGASGAHAVTLEQ SfV1 EVAHASEKYENGKARAIYGVEPMHYVINTYATKGFEERL-HLLPGLEKGASGSQACALEV CVTV IKATASEKFEMGKSRAIYGTGIKDYSIHAYVLADIEPNL-SRVEGIEYGLRGKAMLATMI SV IKATASEKFEMGKARAIYGTGWDYSVHSYALEGVEVNL -SRVDGIEYGLSGKAVLATMI KV IDAVGSEKFEMGKARAIYGTKPIEYCITSYVLDGLEDTL-YKVPGVEGGLTGLDCLSSMI HTV10 IQATASEKYEMGKARAIYGTEPVDYAISSYVLDDIEDHL - YNVDGIESGLTDMDFLVAMI PIRV-3 LHSRHATKFEPGKKRALWNTGLMHYSSSSVLLEQFANNERKNVPWYMPSHTSAFSTAQDF BlaFV1 LHSRFAVKYENEKNRALWNTSIVFYIIGSYLLHAGESRL-DGEDWCDMNAPSGARLADW

$$
\text { : * :***. * * * }
$$

II

PplRV1a IML-ATSDSDTPTWMYDYSDFNINHGLGIQRYIYLRCGTIMAAHGED---HRARSDATAA WIV28 TRMDQLEGR --FGWSYDYKSFENNHTFLDMSNWWNKVSATLAARAQDAGRGDVASDTREV CtV1 ARANITADPDIECSMFDYADFNRHHTPEAQAIVFDVFAELGQAKGA - . - NPDWVRA SfV1 RRALITRDPDLECTMLDYADFNRHHTPEAQSLLFDVFARLGKTKGA CVTV RRMAEVMDRDPECTNIDYADFNYQHTLLAQAATYFALAAVLKNAGH-...-HPDKVKA SV RRMAWTGTAAECTNVDYADFNYQHTLGAQAAVYEELAKSLALAGW-.... - HEDKVKA KV RRKHISQSDSVECTMLDYADFNYQHTMEAQSMVFDTLAEVLGERNS -...... NADKVKA HTV10 RRLRKVESSEVECTMIDYSDFNYQHTLEAQSLVFKCLSARLRTMGH - . - . - HDDKVAA PIRV-3 VRV-RTLR-ERIGVMWDFSDFNINHLLEHMAYLYKRAGEILVERNVS- - GKGDSDVLAA BlaFV1 RRI-QTLIDGKDLLAWDYADFNLNHELQTQCKLWRSYTRGVMHRNDN - - -PSTNALLGRC 190 441 750 762 442 478 69 449 465 379

Figure 2. Cont. 
III

PplRV1a AVWLAQALDNTWLQI-PGQ-ADGKMYRGMLTGIRGTAFFNTLLNVCYAHTVRRNAQELLG WiV28 GQWLAKAFNNVQLRQMGTDMPYHKTNFGLPTGTRGTAFMNTLLNKVYCTLAETFLRTGMD CtV1 NQWVAQAKRSMYTQLPDRE-GRHRVYQGMFSGTRSTDLLNTLLNLAYFRVARRYVDEQLG SfV1 NLWVARAKKQLWALFPGETEP-RKVRQGMFSGTRSTDLINTLLNLAYFEMAKEWLEVQCG CVTV ANWCARSLLNQWVKFPELRTGYVVSQGMFSGFRGTNFLNTILNVAYFRLADKQVRKYFG SV AEWCRDSLLKQYVKFPELRTGYIAVSQGMFSGFRGTNFLNTLANVAYFRVAARHALSLFG KV ANWVSKGLLNQWCKFPNSKGPALRVTQGMFSGCRGTNFINTLLNLTYFQLSSGQVQESLG HTV10 CDWVAAAMLNQWCKFPGVKRVAQRWVGMFSGVRGTNFINTTLNVGYYRVARRWVSENLN PIRV-3 AKWVAASTMETWLEDAESG-VTAKVKRSLMTGVRGTSFVNTILNHVYTEMAREYALRLGG BlaFV1 CKWMCTALENTILEDVTTS-LLIKVSRSMMTGTRGTAFTNTLLNGVYGKLINRNLLSLCG $*$. . .: :**.*:***** ${ }^{*}$

PplRV1a LDPI-VDYRGRIGDIWSTLRSAVYGPFLTDLMLVMGLAGQDSKILYGHGEGEFLRKDYRD

WiV28 V--NALGWGHMGDDVIAATDDYVTANALTMIMSNMGLMTNDLKTWWYEGGELLRKWYTA

CtV1 VHAIDMYHVHQGDDVWVSNRPTWARALFYLNNMGFIFQKSKQMFGVGRGEYLRVYSS

SFV1 LAPTQLYHVHQGDDVWVSNGNPLWARALYYCLNNMGFIFQGSKQMFGPGRGEYLRVLYSG

CVTV VFSRDLYHIHMGDDWISNMCRLWAVALFNCMIAAGLVFQPSKQMFERSKGEFLRWYTK

SV LQPENLYHSHLGDDVFIANKSRLWAWFFNCMSATGLVFQPAKQMFEVSKGEFLRWYTE

KV IRPSALYNIHQGDDWITNESRWWAMALFNHMESCGFVFQASKQMFDTNRGEFLRWYTK

HTV10 LRPTDEFNLHQGDDWISNKSRLWAMAMYESMQSTGLIFQPSKQMFDTSRGEFLRWYTS

PIRV-3 VQLLNDPTYAFGDDVFSSADSHAKAVLFCHVMNTLGTAGTTYKINLE - - LGELLRVSYDE

BlaFV1 EQLFIEPLTGRGDDMISGLSDALIGACGAKLINMMGFAGQEVKVLMGN-VGEFLRQHYTK

\begin{tabular}{llc} 
& \multicolumn{2}{c}{ VI } \\
\cline { 3 - 3 } PplRV1a & G-LCRGFLLRTVPNAVSG & 380 \\
WiV28 & GVGVKGYLNMLPNVCCG & 635 \\
CtV1 & G-HGYGYLGRIANYILR & 939 \\
SfV1 & G-RARGYFARSLANYLLR & 951 \\
CVTV & E-GCRGYLARKIATMVMK & 632 \\
SV & E-GCQGYVARKVATFIMK & 668 \\
KV & E-GCRGYLARAVATTIMK & 259 \\
HTV10 & K-GCQGYLARAVGTLIVK & 639 \\
PiRV-3 & T-GFSGYPLRALVGLVSG & 654 \\
BlaFV1 & D-GVGGYPARACAGLVAG & 580 \\
& \multicolumn{2}{c}{ *: * * }
\end{tabular}

Figure 2. Alignment of amino acid sequence of Phytophthora pluvialis RNA virus 1 genotype a (PplRV1a) putative RNA-dependent RNA polymerase (RdRp) with the RdRp regions of viruses with predicted similarities. Orange boxes indicate the conserved motifs in the viral RdRps. Asterisk indicates positions which have a single, fully conserved residue. Colon indicates conservation between groups of strongly similar properties. Period indicates conservation between groups of weakly similar properties. Numbers indicate the length of amino acid sequence. WiV28: Wuhan insect virus 28 (accession: YP_009342430); CtV1: Circulifer tenellus virus 1 (accession: YP_003800003); SfV1: Spissistilus festinus virus 1 (accession: YP_003800001); CvTV: Culex vishnui subgroup totivirus (accession: BBQ05098); SV: Stinn virus (accession: QRW41701); KV: Koroku virus (accession: QRW41695); HTV10: Hubei toti-like virus 10 (accession: YP_009336493); PiRV-3: Phytophthora infestans RNA virus 3 (accession: YP_009551328); BlaFV1: Bremia lactucae associated fusagravirus1 (accession: QIP68009).

\subsection{PplRV1 Variants and P. pluvialis Cluster NZ1 and NZ2}

PplRV1 from 23 P. pluvialis isolates were fully/near completely sequenced. Except PplRV1_3052 and PplRV1_4018, PplRV1 from NZFS 4015, NZFS 4016 and NZFS 4019 were also fully sequenced. The genome size of PplRV1_4015, PplRV1_4016 and PplRV1_4019 is 6758, 6758 and $6756 \mathrm{nt}$, respectively. The near-complete genomes of other PplRV1 genotypes are about $6500 \mathrm{nt}$ (Table 2). 
Table 2. A list of information of Phytophthora pluvialis RNA virus 1 genotype a (PplRV1a) and Phytophthora pluvialis RNA virus 1 genotype $b$ (PplRV1b) sequences obtained from $P$. pluvialis cluster NZ1 and NZ2 isolates, respectively. Viral sequences were obtained by primer walking and dsRNA sequencing. PplRV1_3052 and PplRV1_4018 are the reference for PplRV1a and PplRV1b genotype viruses, respectively. The full length of reference genomes was determined by $5^{\prime}$ rapid amplification of cDNA ends ( $5^{\prime}$ RACE). Viruses were named corresponding to their P. pluvialis host names. For example, PplRV1_3052 was derived from the P. pluvialis isolate NZFS 3052.

\begin{tabular}{|c|c|c|c|c|c|c|c|}
\hline Virus Name & $\begin{array}{l}\text { GenBank } \\
\text { Accession }\end{array}$ & Genotype & $\begin{array}{l}\text { Length } \\
\text { (nt) }\end{array}$ & GC\% & $\begin{array}{l}\text { Number of } \\
\text { Predicted } \\
\text { RdRp ORFs }\end{array}$ & $\begin{array}{l}\text { Identical Sites } \\
\text { Compared with } \\
\text { PplRV1_4018 }\end{array}$ & $\begin{array}{c}\text { Identical Sites } \\
\text { Compared with } \\
\text { PplRV1_3052 }\end{array}$ \\
\hline PplRV1_3052 & OL799269 & PplRV1a & $6742 *$ & 55.4 & 1 & $92.3 \%$ & - \\
\hline PplRV1_3046 & OL799282 & PplRV1a & 6508 & 55.7 & 1 & $92.0 \%$ & $99.0 \%$ \\
\hline PplRV1_3132 & OL799274 & PplRV1a & 6529 & 55.6 & 1 & $92.2 \%$ & $98.9 \%$ \\
\hline PplRV1_3440 & OL799283 & PplRV1a & 6496 & 55.8 & 1 & $92.0 \%$ & $98.9 \%$ \\
\hline PplRV1_3447 & OL799284 & PplRV1a & 6604 & 55.4 & 1 & $93.2 \%$ & $97.8 \%$ \\
\hline PplRV1_3564 & OL799285 & PplRV1a & 6578 & 55.7 & 1 & $92.1 \%$ & $98.7 \%$ \\
\hline PplRV1_3608 & OL799275 & PplRV1a & 6541 & 55.7 & 1 & $92.1 \%$ & $98.9 \%$ \\
\hline PplRV1_3619 & OL799286 & PplRV1a & 6583 & 55.6 & 1 & $92.3 \%$ & $99.1 \%$ \\
\hline PplRV1_3635 & OL799288 & PplRV1a & 6544 & 55.6 & 1 & $92.1 \%$ & $99.1 \%$ \\
\hline PplRV1_3894 & OL799276 & PplRV1a & 6512 & 55.6 & 1 & $92.2 \%$ & $98.9 \%$ \\
\hline PplRV1_3990 & OL799290 & PplRV1a & 6566 & 55.7 & 1 & $92.0 \%$ & $98.2 \%$ \\
\hline PplRV1_4021 & OL799291 & PplRV1a & 6564 & 55.7 & 1 & $92.2 \%$ & $98.8 \%$ \\
\hline PplRV1_4018 & OL799270 & PplRV1b & $6760 *$ & 55.2 & 2 & - & $92.3 \%$ \\
\hline PplRV1_3000 & OL799281 & PplRV1b & 6597 & 55.6 & 1 & $99.4 \%$ & $92.3 \%$ \\
\hline PplRV1_3632 & OL799287 & PplRV1b & 6557 & 55.6 & 1 & $99.4 \%$ & $92.4 \%$ \\
\hline PplRV1_3880 & OL799289 & PplRV1b & 6563 & 55.6 & 1 & $99.5 \%$ & $92.5 \%$ \\
\hline PplRV1_3998 & OL799277 & PplRV1b & 6545 & 55.6 & 1 & $99.2 \%$ & $92.4 \%$ \\
\hline PplRV1_4014 & OL799278 & PplRV1b & 6677 & 55.5 & 2 & $99.4 \%$ & $92.4 \%$ \\
\hline PplRV1_4015 & OL799271 & PplRV1b & 6758 * & 55.3 & 2 & $99.5 \%$ & $92.3 \%$ \\
\hline PplRV1_4016 & OL799272 & PplRV1b & $6758 *$ & 55.2 & 2 & $99.3 \%$ & $92.2 \%$ \\
\hline PplRV1_4017 & OL799279 & PplRV1b & 6658 & 55.6 & 2 & $99.5 \%$ & $91.2 \%$ \\
\hline PplRV1_4019 & OL799273 & PplRV1b & $6756^{*}$ & 55.3 & 2 & $99.3 \%$ & $92.2 \%$ \\
\hline PplRV1_4234 & OL799280 & PplRV1b & 6554 & 55.6 & 2 & $99.5 \%$ & $92.5 \%$ \\
\hline
\end{tabular}

${ }^{*}$ Full length as determined through $5^{\prime}$ RACE.

The data indicate two distinct PplRV1 variants associated with the two P. pluvialis New Zealand clusters. Comparison of the PplRV1 sequences purified from NZ1 P. pluvialis to the sequences isolated from NZ2 P. pluvialis showed the highest identity correlating with the P. pluvialis host cluster. NZ1-derived PplRV1 sequences are most like the NZ1 PplRV1 reference genome PplRV1a (97.8-99.1\%) rather than the NZ2 PplRV1-derived reference genome PplRV1b (92.0-93.2\%). Conversely, NZ2-derived PplRV1 sequences are most similarity to those of PplRV1b (99.2-99.5\%) rather than PplRV1a (91.2-92.5\%) (Table 2). The data indicate two distinct PplRV1 genotypes ( $a$ or $b$ ), each associated with only one of the two P. pluvialis New Zealand clusters (NZ1 or NZ2, respectively). The intra-variation and inter-variation between PplRV1 genotypes validate the link between the host clusters and virus genotypes.

A phylogenetic analysis also supports that NZ1-associated PplRV1a sequences and NZ2-associated PplRV1b sequences are clustered in two different groups, which has a $100 \%$ support for the grouping (Figure 3). In the PplRV1a group, several sub-groups are evident; the virus from NZFS 3477 is distinctive from other PplRV1a genotype viruses, as other PplRV1a genotype viruses are grouped in the same clade with $100 \%$ support. In the second class of the PplRV1a group, PplRV1_3619 is separated from other viruses, with a support of $90 \%$. Furthermore, in the third class of the PplRV1a group, PplRV1_3052 has a $100 \%$ support for its grouping from other viruses. The rest of PplRV1a genotype viruses, PplRV1_3046, PplRV1_3440, PplRV1_3635, PplRV1_4021, PplRV1_3608, PplRV1_3894, PplRV1_3564, PplRV1_3990 and PplRV1_3132 cluster together, with a support of 17\% 
(Figure 3). In the PplRV1b group, the sequence difference is smaller than in the PplRV1a genotype viruses, as the first sub-group with PplRV1_3998 alone has only 34\% support for the clustering (Figure 3). The seven PplRV1b genomes with a split RdRp (PplRV1_4014, PplRV1_4015, PplRV1_4016, PplRV1_4017, PplRV1_4018, PplRV1_4019, and PplRV1_4234) do not aggregate together tightly in the phylogenetic tree and are distributed amongst the other four genotype $b$ isolates that are predicted to have a single RdRp ORF. Despite the presence of an additional nucleotide resulting in putative split RdRp in seven of the eleven PplRV1b genomes, the distribution of the PplRV1a and PplRV1b variants exactly mirrors the geographical distribution of the host P. pluvialis in New Zealand.

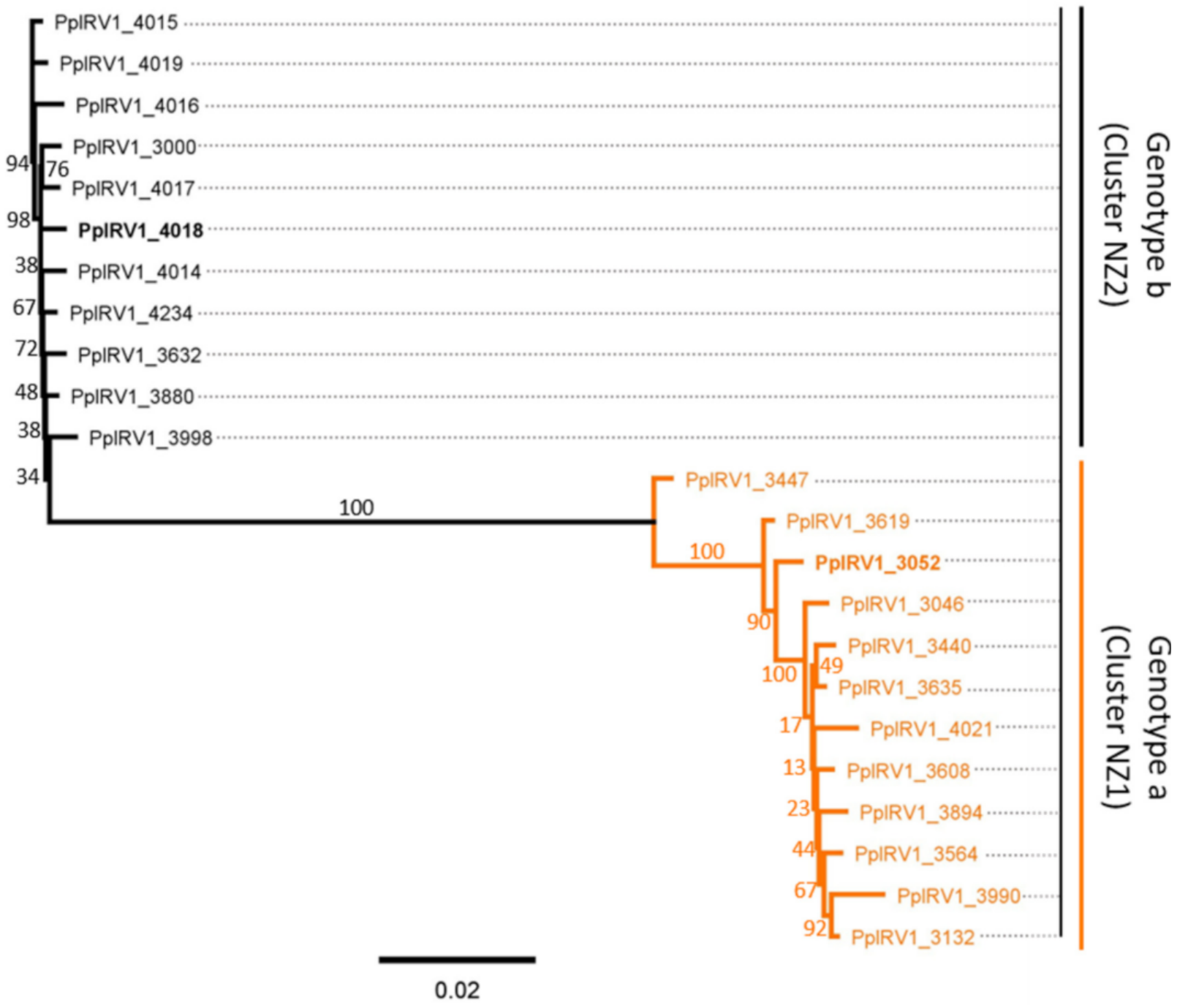

Figure 3. Maximum-likelihood phylogenetic tree based on the core genome alignment of Phytophthora pluvialis RNA virus 1 (PplRV1) sequences isolated from cluster NZ1 and NZ2 Phytophthora pluvialis isolates. The length of the core genome is $6512 \mathrm{nt}$, which was obtained by eight sequencing primer pairs (see Table S2 for details). Black color-coding viruses were isolated from cluster NZ2 P. pluvialis (PplRV1 genotype b). PplRV1_4018 is the reference for NZ2 P. pluvialis viruses. Orange color-coding viruses were isolated from cluster NZ1 P. pluvialis (PplRV1 genotype a). PplRV1_3052 is the reference for NZ1 P. pluvialis viruses. Numbers above the branches show bootstrap support in percentiles in 1000 replicates. Scale bar indicates substitutions per site.

\subsection{Diagnostics of PplRV1 in Phytophthora}

To facilitate further studies of PplRV1, a qPCR diagnostic for the rapid detection and quantification of the virus was developed. Based on the genome comparisons, the ORF1 
region of PplRV1_4018 was chosen as a target as it is the common part of the virus genome, and generic for both PplRV1a and PplRV1b genotype viruses. Furthermore, a multiplex assay that targets the Phytophthora trnM-trnP-trnM region was developed [37]. This host gene target is specific for the genus Phytophthora and allows the simultaneous detection and quantification of the host.

Using a plasmid bearing the target sequences, the limit for virus detection was determined as below 10 copies, and the limit for host detection was established as fewer than 100 copies. The specificity and sensitivity of the assay were established using a panel of 35 Phytophthora/Nothophytophthora isolates (Table S4). Of these, 25 were P. pluvialis bearing PplRV1, and ten were other Phytophthora/Nothophytophthora species not bearing the virus. According to this panel, the specificity and the sensitivity were both $100 \%$, meaning that across the panel no false positives or false negatives were observed.

\section{Discussion}

\subsection{Brief Summary of Findings}

A novel RNA virus designated as PplRV1 was isolated from P. pluvialis in New Zealand. The PplRV1 reference genome encodes two putative proteins, a hypothetical protein and an RdRp. No homology to virus encoded coat proteins was identified within the PplRV1 genome. There are two distinct PplRV1 genotypes: the genotype associated exclusively with P. pluvialis cluster 1 designated as PplRV1a, and the genotype associated exclusively with P. pluvialis cluster 2, PplRV1b. PplRV1a RdRp is predicted to be encoded by a single protein, while the reference genome for the PplRV1b genotype (PplRV1_4018) has the putative RdRp split between ORF2a and ORF2 $b$ that is conserved in seven of eleven genotype $b$ isolates. The qPCR multiplex diagnostic developed for the rapid detection and quantification of both the virus and host revealed that all $P$. pluvialis isolates tested $(n=25)$ were infected with PplRV1.

\subsection{Further Investigation of Genomic Diversity}

As reported previously, the genetic diversity of $P$. pluvialis population shows that $P$. pluvialis cluster 1 (carrying PplRV1a) was the initial introduction into New Zealand [10]. Although the genome of $P$. pluvialis cluster 2 is diverse from that of cluster 1 , the phylogenetic analysis revealed that all P. pluvialis cluster 1 and cluster 2 isolates were grouped in a single terminal clade, which indicates there was only a single incursion of $P$. pluvialis into New Zealand [10]. Since RNA virus replication has high mutation rates, dynamic mutant swarms, known as "viral quasispecies" can form within the host population [38]. PplRV1 may have diverged within New Zealand. This PplRV1 genetic diversity may maintain viral fitness to adapt to genomic variations of its host or the environment, which could explain why some PplRV1b isolates have their putative RdRp split between ORF2a and ORF2b as depicted in Figure 1 for PplRV1_4018.

A -1 frameshifting potentially occurs in a "slippery" overlap sequence between ORF1 and ORF2 in PplRV1. Two stem-loop structures were also predicted downstream of this slippery sequence, which supports the possibility of ribosomal frameshifting in the progress of PplRV1 polyprotein translation. Stem-loops relevant to slippery sequences are also known as "stimulatory" RNA secondary structures, and are able to modulate the -1 frameshift efficiency [39]. Therefore, the stem-loop structures in slippery sequence may play an important role in the control of PplRV1 replication efficiency. For PiRV-3, an in-depth analysis of the translational scenario showed that a slippery sequence caused a -1 frameshift between ORF1 and ORF2, thereby enabling the expression of a fusion protein [17]. The structure of PplRV1_3052 (genotype a) points to an analogous mechanism that allows either the translation of an ORF1-ORF2 fusion protein, or additionally the possibility of translation of ORF1 by itself. In PplRV1_4018 (and several other genotype $\mathrm{b}$ isolates), the situation is less clear, owing to a single nucleotide insertion at position 5064 within ORF2. The described mechanism of a -1 frameshift would in this case lead to an ORF1-ORF2a fusion protein. ORF2b, on the other hand, is encoded in the same 
reading frame as ORF1, and contains several of the critical residues for RdRp function. It remains unclear if a regulation of the -1 frameshift would allow the translation of an ORF1-ORF2b fusion protein, which then complements an ORF1-ORF2a fusion protein into a functional RdRp, or if a different, so far unidentified mechanism allows the translation of this ORF. Additional research is necessary to elucidate this enigma, and to understand its evolutionary history and impact.

A BLAST search using the PplRV1 ORF2 sequence revealed that it has similarity to PiRV-3, totiviruses and the RdRps of unclassified RNA viruses. However, phylogenetic analysis of PplRV1 RdRps and these related viral RdRps found PplRV1 does not cluster with any of those viruses (Table 1). Moreover, protein alignment between the RdRps of PplRV1 and its relevant viruses found PplRV1 shares very low similarity with them (Table 1). In fact, all other viruses are more closely related to each other than any of them is to PplRV1. Therefore, PplRV1 may represent the first member of a new RNA virus family.

\section{3. qPCR Multiplex Diagnostic}

In this study, a diagnostic method using qPCR technique has been developed, which can accurately and quantitatively detect PplRV1 and Phytophthora using a single sample. Compared with the traditional diagnostic method of using standard PCR, our diagnostics can be used to determine the titers of PplRV1 by using an appropriate standard. For that purpose, the target regions of the assay were placed on a plasmid, and appropriate dilutions were created to represent specific copy numbers.

We detected PplRV1 exclusively in P. pluvialis and not in other Phytophthora/Nothophytophthora species pure cultures so far, despite ten isolates being from other species. It would be beneficial to investigate a broader variety of Phytophthora species and other oomycete species as well as environmental samples, i.e., infected pine needles, and water and soil samples from pine woods. A comprehensive survey of PplRV1 in isolates from North America could shed light on the biology of PplRV1, and potentially on the history of its introduction to New Zealand. With the help of PplRV1 diagnostics described in this work, we can obtain a better understanding of the prevalence and host range of PplRV1.

\subsection{PplRV1 Distribution}

Investigation of PplRV1 (a and b) distribution correlated entirely with the P. pluvialis cluster (1 and 2, respectively), indicating co-evolution of PplRV1 with its host P. pluvialis cluster since its introduction to New Zealand [6]. The result also implies low mobility of PplRV1 between collocated P. pluvialis isolates. The lack of PplRV1 movement between host clusters could be caused by exclusion (i.e., all P. pluvialis in New Zealand carry the virus and an already infected isolate cannot be superinfected by a different virus variant), a lack of a vector for PplRV1 in New Zealand, or the inability for PplRV1 to move by anastomosis between the two P. pluvialis clusters present in New Zealand. It is unlikely that PplRV1 forms a virion and the unencapsulated RNA may be unstable in the environment. Whether the initial introduction of P. pluvialis carried PplRV1, or if this association occurred shortly after entry into New Zealand, will require further research to confirm. Importantly, research is needed to survey the distribution of PplRV1 in North America, and to determine its genomic features. In addition, determining whether PplRV1 is present in other Phytophthora species within New Zealand, the USA and other countries, especially those growing Pseudotsuga menziesii and Pinus radiata, would provide a clearer picture of its worldwide distribution and introduction history into New Zealand.

\subsection{Future Research}

The unique genetic structure of some PplRV1b isolates leads to additional questions, for example, how are ORF2a and ORF2b expressed to form a functional RdRp enzyme? A thorough biochemical investigation of the RdRp enzymes from isolates with the different genome structures could illuminate the function and potential evolutionary relevance of the different genome organizations described in this work. 
An analysis of the presence of PplRV1 in P. pluvialis strains isolated in North America, and the comparison of their genomes with the ones described here, will provide further insight into the function of the virus.

The significance of PplRV1a and b for its host remains unclear. To date, no P. pluvialis isolate without one of the PplRV1 virus variants has been observed. The generation of such a PplRV1-free isolate will be crucial for future work to understand the ecological and evolutionary roles of the virus.

Some mycoviruses affect the host virulence and may play an important role in the development of the controlling methods for plant diseases. For example, CHV1 decreases the pathogenicity of its host $C$. parasitica which was used as a biocontrol agent of chestnut blight in Europe [21]; Conversely, PiRV-2 stimulates the sporangia production in P. infestans and could impact the ecological fitness of $P$. infestans [23]. Since $P$. pluvialis results in severe red needle cast disease in New Zealand, future research on the virus transmission and whether PplRV1 affects host virulence (i.e., hyper- or hypo-virulence) will be important for the development of a potential biocontrol for red needle cast disease.

Supplementary Materials: The following supporting information can be downloaded at: https: / www. mdpi.com/article/10.3390/v14020247/s1, Figure S1: Agarose-gel electrophoresis of PplRV1_4018 dsRNA RT-PCR products; Table S1: Phytophthora pluvialis isolates used for virus genome characterisation in this study; Table S2: A list of primers used for Phytophthora pluvialis RNA virus 1 (PplRV1) dsRNA sequencing and PplRV1 detection and diagnostics; Table S3: The optimized reaction of RT-qPCR for Phytophthora pluvialis RNA virus 1 detection; Table S4: A panel of Phytophthora/Nothophytophthora isolates used for the virus screen, including 25 Phytophthora pluvialis isolates, nine other Phytophthora species/isolates and one Nothophytophthora species.

Author Contributions: Conceptualization, Z.X., F.K., G.R.S., M.E.K., R.A.F., R.L.M. and R.M.M.; Methodology, Z.X., F.K., G.R.S., M.E.K., R.A.F. and R.L.M.; Formal analysis, Z.X.; Investigation, Z.X. and M.E.K.; Resources, F.K. and R.L.M.; Data curation, Z.X. and R.A.F.; Writing-Original Draft Preparation, Z.X., F.K. and R.A.F.; Writing-Review and Editing, Z.X., F.K., G.R.S., M.E.K., R.A.F., R.L.M. and R.M.M.; Visualization, Z.X., F.K. and R.A.F.; Supervision, F.K., G.R.S., R.A.F., R.L.M. and R.M.M.; Project Administration, F.K., G.R.S., R.A.F., R.L.M. and R.M.M.; Funding Acquisition, G.R.S. All authors have read and agreed to the published version of the manuscript.

Funding: This research was funded by the New Zealand Biological Heritage National Science Challenge via grant number 1617-22-009 C.

Institutional Review Board Statement: Not applicable.

Informed Consent Statement: Not applicable.

Data Availability Statement: Data supporting reported results are available from GenBank under accession numbers listed in Table 2.

Acknowledgments: We thank Bevan Weir, Manaaki Whenua-Landcare Research (Auckland, New Zealand) for his support for this research project.

Conflicts of Interest: The authors declare no conflicts of interest. The sponsors had no role in the design, execution, interpretation, or writing of the study.

\section{References}

1. Rossman, A.Y.; Palm, M.E. Why are Phytophthora and other Oomycota not true Fungi? Outlooks Pest Manag. 2006, 17, 217. [CrossRef]

2. Henfling, J.W. Late Blight of Potato; International Potato Center: Lima, Peru, 1987; Volume 4.

3. Beever, R.E.; Waipara, N.W.; Ramsfield, T.D.; Dick, M.A.; Horner, I.J. Kauri (Agathis australis) under threat from Phytophthora. Phytophthoras For. Nat. Ecosyst. 2009, 74, 74-85.

4. Weir, B.S.; Paderes, E.; Anand, N.; Uchida, J.Y.; Pennycook, S.R.; Bellgard, S.E.; Beever, R.E. A taxonomic revision of Phytophthora Clade 5 including two new species, Phytophthora agathidicida and P. cocois. Phytotaxa 2015, 205, 21-38. [CrossRef]

5. Scott, P.; Williams, N. Phytophthora diseases in New Zealand forests. NZ J. For. 2014, $59,15$.

6. Dick, M.A.; Williams, N.M.; Bader, M.K.-F.; Gardner, J.F.; Bulman, L.S. Pathogenicity of Phytophthora pluvialis to Pinus radiata and its relation with red needle cast disease in New Zealand. New Zealand J. For. Sci. 2014, 44, 6. [CrossRef] 
7. Reeser, P.; Sutton, W.; Hansen, E. Phytophthora pluvialis, a new species from mixed tanoak-Douglas-fir forests of western Oregon, USA. North Am. Fungi 2013, 8, 1-8. [CrossRef]

8. Hansen, E.M.; Reeser, P.; Sutton, W.; Gardner, J.; Williams, N. First report of Phytophthora pluvialis causing needle loss and shoot dieback on Douglas-fir in Oregon and New Zealand. Plant. Dis. 2015, 99, 727. [CrossRef]

9. Brar, S.; Tabima, J.F.; McDougal, R.L.; Dupont, P.-Y.; Feau, N.; Hamelin, R.C.; Panda, P.; LeBoldus, J.M.; Grünwald, N.J.; Hansen, E.M.; et al. Genetic diversity of Phytophthora pluvialis, a pathogen of conifers, in New Zealand and the west coast of the United States of America. Plant. Pathol. 2017, 67, 1131-1139. [CrossRef]

10. Tabima, J.F.; Gonen, M.L.; Gómez-Gallego, M.M.; Panda, P.; Grünwald, N.J.; Hansen, E.M.; McDougal, R.; LeBoldus, J.M.; Williams, N.M. Molecular Phylogenomics and Population structure of Phytophthora pluvialis. Phytopathology® 2021, 111, 108-115. [CrossRef]

11. Hacker, C.V.; Brasier, C.M.; Buck, K.W. A double-stranded RNA from a Phytophthora species is related to the plant endornaviruses and contains a putative UDP glycosyltransferase gene. J. Gen. Virol. 2005, 86, 1561-1570. [CrossRef]

12. Kozlakidis, Z.; Brown, N.A.; Jamal, A.; Phoon, X.; Coutts, R.H.A. Incidence of endornaviruses in Phytophthora taxon douglasfir and Phytophthora ramorum. Virus Genes 2009, 40, 130-134. [CrossRef] [PubMed]

13. Poimala, A.; Vainio, E. Complete genome sequence of a novel toti-like virus from the plant-pathogenic oomycete Phytophthora cactorum. Arch. Virol. 2020, 165, 1679-1682. [CrossRef] [PubMed]

14. Shiba, K.; Hatta, C.; Sasai, S.; Tojo, M.; Ohki, S.T.; Mochizuki, T. A novel toti-like virus from a plant pathogenic oomycete Globisporangium splendens. Virology 2019, 537, 165-171. [CrossRef] [PubMed]

15. Cai, G.; Myers, K.; Hillman, B.I.; Fry, W.E. A novel virus of the late blight pathogen, Phytophthora infestans, with two RNA segments and a supergroup 1 RNA-dependent RNA polymerase. Virology 2009, 392, 52-61. [CrossRef]

16. Cai, G.; Myers, K.; Fry, W.E.; Hillman, B.I. A member of the virus family Narnaviridae from the plant pathogenic oomycete Phytophthora infestans. Arch. Virol. 2011, 157, 165-169. [CrossRef]

17. Cai, G.; Krychiw, J.F.; Myers, K.; Fry, W.E.; Hillman, B.I. A new virus from the plant pathogenic oomycete Phytophthora infestans with an $8 \mathrm{~kb}$ dsRNA genome: The sixth member of a proposed new virus genus. Virology 2013, 435, 341-349. [CrossRef]

18. Cai, G.; Myers, K.; Fry, W.E.; Hillman, B.I. Phytophthora infestans RNA virus 2, a novel RNA virus from Phytophthora infestans, does not belong to any known virus group. Arch. Virol. 2018, 164, 567-572. [CrossRef]

19. Liu, H.; Fu, Y.; Li, B.; Yu, X.; Xie, J.; Cheng, J.; Ghabrial, S.A.; Li, G.; Yi, X.; Jiang, D. Widespread horizontal gene transfer from circular single-stranded DNA viruses to eukaryotic genomes. BMC Evol. Biol. 2011, 11, 276. [CrossRef]

20. Márquez, L.M.; Redman, R.S.; Rodriguez, R.J.; Roossinck, M.J. A Virus in a Fungus in a Plant: Three-Way Symbiosis Required for Thermal Tolerance. Science 2007, 315, 513-515. [CrossRef]

21. Robin, C.; Heiniger, U. Chestnut blight in Europe: Diversity of Cryphonectria parasitica, hypovirulence and biocontrol. For. Snow Landsc. Res. 2001, 76, 361-367.

22. Rigling, D.; Prospero, S. Cryphonectria parasitica, the causal agent of chestnut blight: Invasion history, population biology and disease control. Mol. Plant. Pathol. 2017, 19, 7-20. [CrossRef] [PubMed]

23. Cai, G.; Fry, W.E.; Hillman, B.I. PiRV-2 stimulates sporulation in Phytophthora infestans. Virus Res. 2019, 271, 197674. [CrossRef] [PubMed]

24. Ghabrial, S.A.; Caston, J.R.; Jiang, D.; Nibert, M.L.; Suzuki, N. 50-plus years of fungal viruses. Virology 2015, 479-480, 356-368. [CrossRef] [PubMed]

25. Valverde, R.; Nameth, S.; Jordan, R. Analysis of double-stranded RNA for plant virus diagnosis. Plant Dis. 1990, 74, 255-258.

26. Ristaino, J.B.; Ivors, K.; Bonants, P.; Alpizar, L.; Mónica, B.; Meneses, M. Rapid diagnostic tools for Phytophthora on horticultural crops. In Workshop: Implementación de herramientas de Diagnóstico Rápido Para Phytophthora en Cultivos Agrícolas en Centro América; Universidad de Costa Rica: San José, CA, USA, 2010.

27. Katoh, K.; Standley, D.M. MAFFT multiple sequence alignment software version 7: Improvements in performance and usability. Mol. Biol. Evol. 2013, 30, 772-780. [CrossRef]

28. Katoh, K.; Misawa, K.; Kuma, K.I.; Miyata, T. MAFFT: A novel method for rapid multiple sequence alignment based on fast Fourier transform. Nucleic Acids Res. 2002, 30, 3059-3066. [CrossRef]

29. Guindon, S.; Dufayard, J.-F.; Lefort, V.; Anisimova, M.; Hordijk, W.; Gascuel, O. New Algorithms and Methods to Estimate Maximum-Likelihood Phylogenies: Assessing the Performance of PhyML 3.0. Syst. Biol. 2010, 59, 307-321. [CrossRef]

30. Guindon, S.; Gascuel, O. A simple, fast, and accurate algorithm to estimate large phylogenies by maximum likelihood. Syst. Biol. 2003, 52, 696-704. [CrossRef]

31. Lu, S.; Wang, J.; Chitsaz, F.; Derbyshire, M.K.; Geer, R.C.; Gonzales, N.R.; Gwadz, M.; Hurwitz, D.I.; Marchler, G.H.; Song, J.S.; et al. CDD/SPARCLE: The conserved domain database in 2020. Nucleic Acids Res. 2020, 48, D265-D268. [CrossRef]

32. Madeira, F.; Park, Y.M.; Lee, J.; Buso, N.; Gur, T.; Madhusoodanan, N.; Basutkar, P.; Tivey, A.R.N.; Potter, S.C.; Finn, R.D.; et al. The EMBL-EBI search and sequence analysis tools APIs in 2019. Nucleic Acids Res. 2019, 47, W636-W641. [CrossRef]

33. Moon, S.; Byun, Y.; Han, K. FSDB: A frameshift signal database. Comput. Biol. Chem. 2007, 31, 298-302. [CrossRef] [PubMed]

34. Untergasser, A.; Cutcutache, I.; Koressaar, T.; Ye, J.; Faircloth, B.C.; Remm, M.; Rozen, S.G. Primer3-New capabilities and interfaces. Nucleic Acids Res. 2012, 40, e115. [CrossRef] [PubMed]

35. Koressaar, T.; Remm, M. Enhancements and modifications of primer design program Primer3. Bioinformatics 2007, 23, 1289-1291. [CrossRef] [PubMed] 
36. Bruenn, J. A structural and primary sequence comparison of the viral RNA-dependent RNA polymerases. Nucleic Acids Res. 2003, 31, 1821-1829. [CrossRef]

37. Bilodeau, G.J.; Martin, F.N.; Coffey, M.D.; Blomquist, C.L. Development of a Multiplex Assay for Genus- and Species-Specific Detection of Phytophthora Based on Differences in Mitochondrial Gene Order. Phytopathology® 2014, 104, 733-748. [CrossRef]

38. Domingo, E.; Holland, J.J. RNA virus mutations and fitness for survival. Annu. Rev. Microbiol. 1997, 51, 151-178. [CrossRef]

39. Giedroc, D.P.; Theimer, C.A.; Nixon, P.L. Structure, stability and function of RNA pseudoknots involved in stimulating ribosomal frameshifting. J. Mol. Biol. 2000, 298, 167-185. [CrossRef] 